\title{
Determinants of early breastfeeding initiation and exclusive breastfeeding in Colombia
}

\author{
Sheridan Finnie*, Rafael Peréz-Escamilla and Gabriela Buccini \\ Yale School of Public Health, Yale University, 60 College Street, New Haven, CT06520, USA
}

Submitted 14 June 2018: Final revision received 19 April 2019: Accepted 9 May 2019: First published online 7 October 2019

\begin{abstract}
Objective: To identify modifiable risk factors associated with early initiation of breastfeeding (EIBF) and exclusive breastfeeding (EBF) in Colombia.

Design: Cross-sectional study from the 2010 Colombia nationally representative Demographic Health Survey (DHS). Studied exposures were categorized into five hierarchical blocks of increasing proximity to the outcomes: household, maternal, health systems, child, and early feeding characteristics. The two outcomes examined were delayed breastfeeding initiation among infants $<24$ months and interruption of EBF among infants $<6$ months. Prevalence ratios were computed using Poisson regression analysis with robust error variance, adjusted for sampling weights, following a hierarchical modelling approach.

Setting: Nationally representative cross-sectional survey from Colombia.

Participants: The EIBF analytical sample included 6592 and the EBF sample 1512 women with young children.

Results: EIBF prevalence was $65.6 \%$ in children under 24 months and EBF was $43 \%$ in infants under 6 months. Modifiable risk factors associated with delayed breastfeeding initiation were: C-section $(\mathrm{PR}=2 \cdot 08$, CI $95 \%=1 \cdot 92,2 \cdot 25)$, maternal overweight/obesity (PR $=1.09$, CI $95 \%=1 \cdot 01,1 \cdot 17)$, lack of skilled attendant at birth $(\mathrm{PR}=1 \cdot 09$, CI $95 \%=1 \cdot 01,1 \cdot 18)$. Modifiable risk factors for EBF interruption were $\mathrm{C}$-section $(\mathrm{PR}=1 \cdot 12$, CI $95 \%=1 \cdot 02,1.23)$ and prelacteal feeding ( $\mathrm{PR}=1.51$, CI $95 \%=1.37,1.68)$. Non-pregnancy intention was a protective factor for EBF interruption ( $\mathrm{PR}=0.82$, CI $95 \%=0.72,0.93)$.

Conclusions: C-section, lack of skilled attendant at birth, prelacteal feeding, maternal nutritional status, and pregnancy intention were modifiable factors associated with suboptimal breastfeeding practices in Colombia.
\end{abstract}

\author{
Keywords \\ Breastfeeding \\ Exclusive breastfeeding \\ Infant and young child feeding \\ Early breasffeeding initiation
}

Investing in breastfeeding protection, promotion, and support programmes is important to promote optimal child health and child development throughout the lifespan, including maternal health, and foster economic growth ${ }^{(1,2)}$.

Since the early 1990s, Colombia has identified breastfeeding and child welfare programmes as key strategies to reduce infant mortality and morbidity as well as to improve child developmental potential ${ }^{(3)}$. The enabling of a breastfeeding friendly environment began in 1992 with the development of the Breastfeeding Support National Plan and three central policies that endorsed the WHO International Code of Marketing of Breastmilk Substitutes (WHO's Code), the Baby Friendly Hospital Initiative (BFHI) and the formation of a National Breastfeeding Committee; however, oversight and regulatory responsibility for these policies were not granted to a specified entity ${ }^{(4,5)}$. Since then, the National Breastfeeding Committee has disbanded and BFHI implementation remains limited. Of the 51430 private and public health institutions licensed to provide maternal and child care in 2009, only 330 (0.64\%) were accredited as $\mathrm{BFHI}^{(3,6,7)}$. This is not surprising given that that the country lacks a centralized coordinating entity empowered to recommend and implement needed breastfeeding policies and programmes $^{(8-10)}$.

Primary indicators for assessing in-country breastfeeding practices include early initiation of breastfeeding (EIBF) and exclusive breastfeeding (EBF). EIBF has been shown to be associated with positive infant morbidity and mortality outcomes, associated with colostrum feeding and overall duration of breastfeeding ${ }^{(1,6)}$. In Colombia, breastfeeding practices have not improved over the years. 
Indeed, early breastfeeding initiation rates decreased from $61 \%$ in 2000 to $48.9 \%$ in 2005 and then partially rebounded to $57 \%$ in 2010. Furthermore, EBF decreased from $47 \%$ in 2005 to $43 \%$ in $2010^{(11-13)}$. To our knowledge, the reasons for this trend have not been studied. In our study we hypothesize that this may be influenced by healthcare system-adjacent modifiable risk factors ${ }^{(3,14)}$ combined with population level factors such as household and maternal characteristics $^{(7,8)}$. There is a dearth of studies investigating the determinants of EIBF and EBF in Colombia. Thus, it is important to identify relevant modifiable risk factors for improving breastfeeding that can be integrated into and delivered through a package of interventions to improve optimal breastfeeding practices more rapidly in Colombia $^{(15)}$. Examples of modifiable risk factors to improve breastfeeding practices through healthcare system interventions are maternal nutrition status, pregnancy intention, type and place of delivery, prenatal and prelacteal feedings. Hence, the primary aim of this study is to identify modifiable risk factors for improving EIBF and EBF among infants under 24 months and 6 months of age, respectively.

\section{Methods}

\section{Study setting}

The study used data from the Colombian Demographic and Health Survey (DHS) conducted from November 2009 through November 2010. Colombia is an upper middleincome country with the fourth highest rate of maternal mortality in South America (92 per 100000 live births) and relatively high rates of infant mortality ( 15 per 1000 live births $)^{(16)}$. Data on maternal and child health and nutrition outcomes were collected in the DHS for children born in the 36 months preceding data collection, and then our analyses subsets were restricted to the relevant age ranges (i.e. born during the previous 24 months for EIBF and during the previous 6 months for EBF analyses). The survey's sampling framework was based on a stratified, multiple-stage cluster sampling design, drawing from national level census data, and regional/household level registries across geographical areas ${ }^{(12)}$.

\section{Outcome variables}

This study has two primary outcomes operationalized based on the following definitions from the WHO Infant and Young Child Feeding (IYCF) guidelines ${ }^{(17)}$.

Early initiation of breastfeeding was defined as the 'proportion of children born in the last 24 months who were put to breast within $1 \mathrm{~h}$ of birth' (18). The outcome variable was delayed breastfeeding initiation, i.e. not being breastfed within the $1 \mathrm{~h}$ of birth.

Exclusive breastfeeding was defined as the "proportion of infants $0-5$ months of age who were fed exclusively with breastmilk' and was based on maternal current status recall in reference to the previous day ${ }^{(18)}$. EBF was categorized based on nineteen questions querying the mother if her child consumed 29 specific food or liquid items other than breastmilk in the previous day. Infants were considered to be exclusively breastfed if breastmilk was the only source of nutrition and hydration, without any additional solid or liquid supplement including water ${ }^{(18)}$. The outcome variable 'EBF interruption' was defined as not being exclusively breastfed in the last $24 \mathrm{~h}$ before the interview.

\section{Independent variables}

The explanatory variables were grouped into four (EIBF model) and five (EBF model) distal to proximal blocks. Both models included household, maternal, health systems, and child characteristics, and the EBF analysis also included the early feeding environment (see conceptual model in Fig. 1). Breastfeeding initiation and exclusivity are influenced by a range of interrelated and temporally distinct factors at different levels of proximity. Conceptual hierarchical frameworks are an appropriate technique to evaluate the individual determinants of health outcomes as outlined by Victora et al. ${ }^{(19)}$. Specifically, hierarchical models have been recommended and applied to study breastfeeding outcomes globally ${ }^{(20-22)}$.

The DHS composite wealth index was used to describe household wealth and is based on a standard list of household assets classified into five wealth quintiles (income quintile variable). The number of adults in the household was estimated by subtracting the number of children living in the residence from the total number of usual household residents plus the number of visitors who slept in the house the previous night. A skilled attendant at birth was affirmed if delivery was performed by a doctor or nurse (yes); and not performed by a skilled attendant if it involved others, including auxiliary nurse and traditional birth attendant (no). DHS anthropometric measurements (height and weight) were assessed directly at the time of the survey using a measuring board and properly calibrated scale. Maternal overweight/obesity was estimated using the standard BMI cut-off points; i.e. $\geq 25$ for overweight and $\geq 30$ for obesity. Newborns were considered to have received prenatal feeds when any one of nineteen questions querying regarding any liquids given to the infant in the first $3 \mathrm{~d}$ post birth, including infant formula and water with or without sugar, were affirmed. Prelacteal feeding was considered to have not occurred only when all nineteen items were not affirmed ${ }^{(23)}$. The number of prenatal visits were considered to be adequate if they were at least four based on Colombia's guidelines ${ }^{(24)}$. Prenatal visits numbering ten or more was considered potentially indicative of a higher risk pregnancy ${ }^{(25)}$.

\section{Analytical sample}

In the EIBF analysis the initial sample included 6694 children under 24 months of age born to women aged 


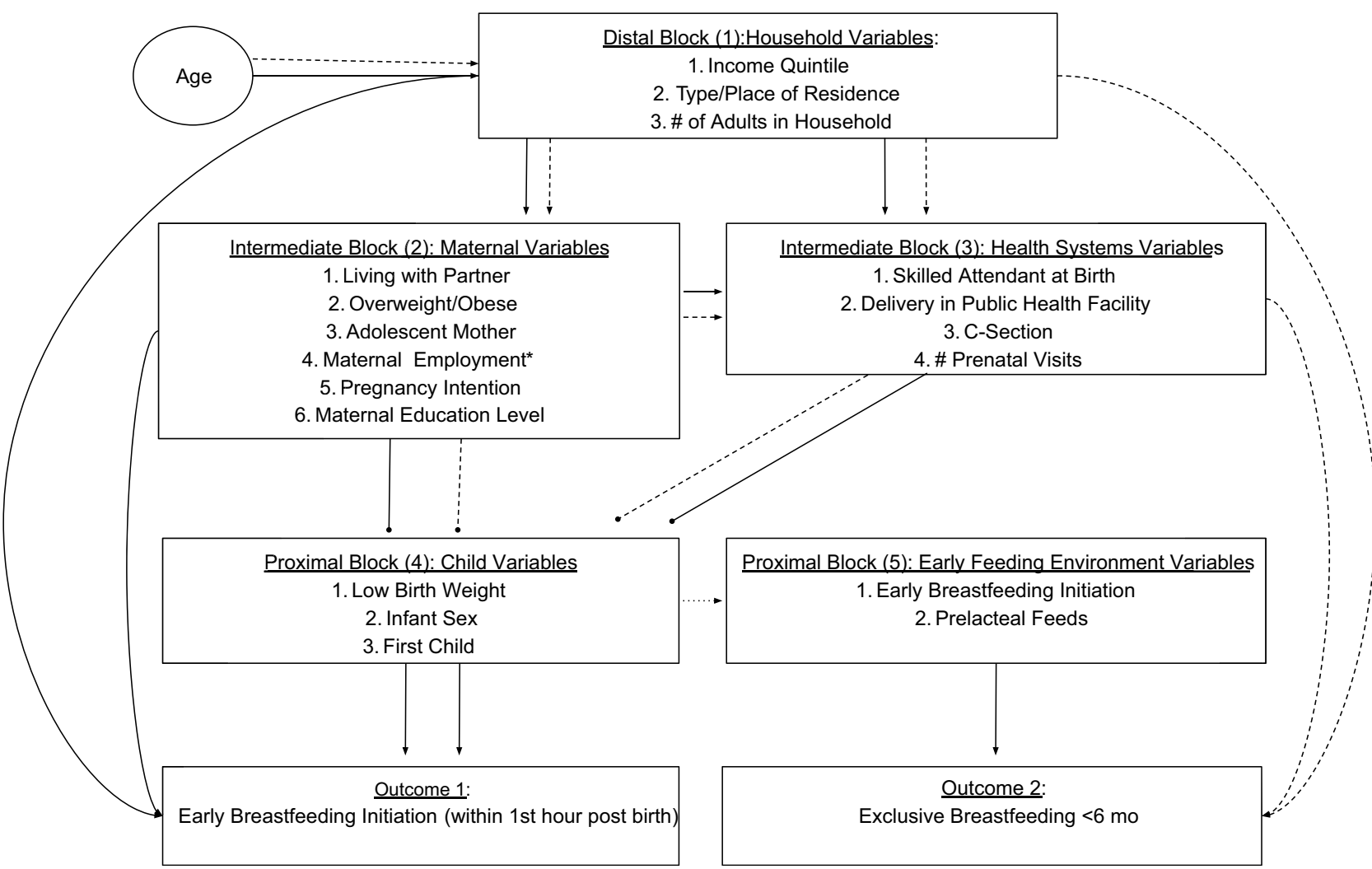

Fig. 1 Conceptual model: description of hierarchical interrelationship between explanatory and outcome variables. ${ }^{\star}$ Maternal Employment variable only considered in outcome 2, exclusive breastfeeding model

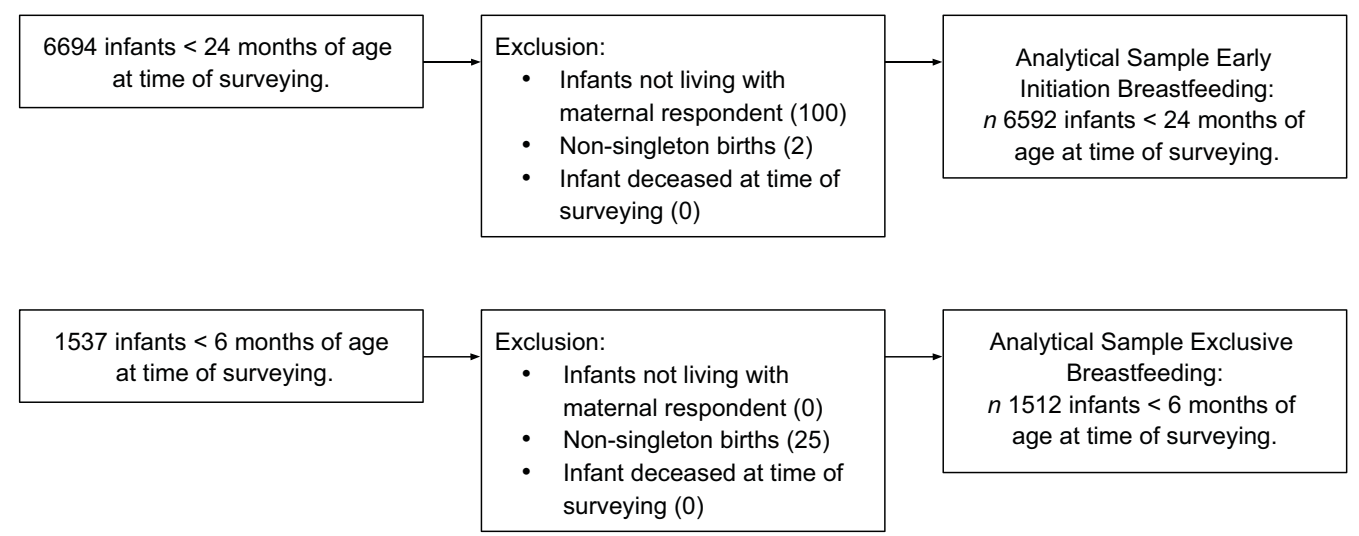

Fig. 2 Flow chart depicting analytical sample selection criteria.

13-49 years old. After excluding multiple births, children who did not live in the maternal household, and nonliving children, the analytical sample included 6592 children born within the 24 months preceding data collection. In the EBF analysis, the initial sample included 1537 infants under 6 months of age. After excluding multiple births, children who did not live in the maternal household, and non-living children the analytical sample included 1512 infants under 6 months of age. Figure 2 presents detailed information regarding analytical sample selection.

\section{Data analyses}

Statistical analyses were performed using STATA version 15.1 (Stata Corp.). Separate modified Poisson logistic regressions with robust error variance weighted by the DHS weight statistic (iweight $=\mathrm{wgt}$ ) for nationally representative proportions were conducted to identify explanatory factors for each outcome. We used the statistical command meglm with (iweight $=$ wgt) option as it is the preferred command to fit mixed-effects generalized linear models to hierarchical datasets with normally distributed effects, such as described in our DHS dataset ${ }^{(26,27)}$. The study was 
Table 1 Descriptive characteristics of analytical sample ( $n 13567)$, and bivariate analyses of delayed breastfeeding initiation among children aged under 24 months and unadjusted prevalence ratios (Demographic Health Surveys, Colombia 2010)

\begin{tabular}{|c|c|c|c|c|c|c|}
\hline & & \multicolumn{2}{|c|}{ Analytical sample } & \multicolumn{3}{|c|}{ Delayed breastfeeding initiation } \\
\hline & & $\%^{*}$ & $n \dagger$ & $\% \ddagger$ & Non-adjusted PR & $95 \% \mathrm{Cl}$ \\
\hline \multirow{2}{*}{ Early breastfeeding initiation (within $1 \mathrm{~h}$ ) } & Yes & $65 \cdot 6$ & 4305 & - & - & - \\
\hline & No & 34.4 & 2056 & - & - & - \\
\hline \multicolumn{7}{|l|}{ Distal block (1): household variables } \\
\hline \multirow[t]{4}{*}{ Place residence } & Urban & $72 \cdot 3$ & 4195 & $35 \cdot 3$ & 1.00 & \\
\hline & Rural & $27 \cdot 7$ & 2397 & $32 \cdot 1$ & 0.86 & $0.80,0.94$ \\
\hline & Poorest & $25 \cdot 2$ & 2448 & $32 \cdot 2$ & 1.00 & \\
\hline & Poorer & $23 \cdot 6$ & 1765 & 35 & $1 \cdot 15$ & $1.04,1.27$ \\
\hline \multirow[t]{4}{*}{ Income quintile } & Middle & $22 \cdot 2$ & 1209 & $36 \cdot 1$ & 1.20 & $1.08,1.34$ \\
\hline & Richer & $17 \cdot 3$ & 740 & 34.5 & 1.22 & $1.08,1.38$ \\
\hline & Richest & 11.6 & 430 & 34.9 & 1.28 & $1.11,1.48$ \\
\hline & $1-2$ & $28 \cdot 3$ & 1797 & 33.2 & 1.00 & \\
\hline \multirow{2}{*}{$\begin{array}{l}\text { Number adults in household } \\
\text { Intermediate block (2): maternal variables }\end{array}$} & $3+$ & 71.8 & 4795 & 34.9 & 0.99 & $0.91,1.07$ \\
\hline & & & & & & \\
\hline \multirow[t]{2}{*}{ Living with partner currently } & Yes & $73 \cdot 1$ & 4931 & $35 \cdot 2$ & 1.00 & \\
\hline & No & 26.9 & 1661 & $32 \cdot 3$ & 0.91 & $0.84,0.99$ \\
\hline \multirow[t]{2}{*}{ Education level } & Higher education & 75.5 & 4587 & $36 \cdot 1$ & 1.00 & \\
\hline & Lower education & 24.5 & 2005 & $29 \cdot 2$ & 0.77 & $0.71,0.84$ \\
\hline \multirow{2}{*}{ Maternal overweight/obese } & No & $59 \cdot 1$ & 3547 & $32 \cdot 7$ & 1.00 & \\
\hline & Yes & 40.9 & 2515 & $34 \cdot 4$ & 1.09 & $1 \cdot 01,1 \cdot 17$ \\
\hline \multirow[t]{2}{*}{ Adolescent mother $(<19)$} & No & 81.9 & 5357 & 34.7 & 1.00 & \\
\hline & Yes & $18 \cdot 1$ & 1235 & 33.0 & 0.97 & $0.88,1.06$ \\
\hline \multirow[t]{3}{*}{ Pregnancy intention } & Then & 47.6 & 3000 & $35 \cdot 1$ & 1.00 & \\
\hline & Later & $30 \cdot 2$ & 1959 & $35 \cdot 8$ & 1.00 & $0.92,1.08$ \\
\hline & No More & $22 \cdot 2$ & 1633 & $31 \cdot 2$ & 0.85 & $0.78,0.94$ \\
\hline \multicolumn{7}{|l|}{ Intermediate block (3): health systems variables } \\
\hline \multirow[t]{2}{*}{ Skilled attendant at birth } & Yes & 41.7 & 2600 & $32 \cdot 3$ & 1.00 & \\
\hline & No & $58 \cdot 3$ & 3992 & 36 & 1.03 & $0.96,1.11$ \\
\hline \multirow{2}{*}{ Delivery in public health facility } & Yes & $95 \cdot 2$ & 5860 & 34.7 & 1.00 & \\
\hline & No & 4.8 & 631 & $29 \cdot 4$ & 0.68 & $0.58,0.80$ \\
\hline \multirow[t]{2}{*}{ C-Section } & No & 62.9 & 4399 & $24 \cdot 6$ & 1.00 & \\
\hline & Yes & $37 \cdot 1$ & 2193 & 51.4 & $2 \cdot 10$ & $1 \cdot 96,2 \cdot 25$ \\
\hline \multirow[t]{3}{*}{ Number prenatal visits } & $4-9$ & 80.7 & 4928 & $34 \cdot 0$ & 1.00 & \\
\hline & $0-3$ & 11.3 & 1126 & 34.0 & 0.82 & $0.74,0.91$ \\
\hline & $10+$ & 8.0 & 371 & 40.5 & 1.25 & $1 \cdot 10,1.42$ \\
\hline \multicolumn{7}{|l|}{ Proximal block (4): child variables } \\
\hline \multirow[t]{2}{*}{ Low birth weight } & No & $91 \cdot 8$ & 4705 & $33 \cdot 3$ & 1.00 & \\
\hline & Yes & $8 \cdot 2$ & 353 & $48 \cdot 7$ & 1.48 & $1.31,1.68$ \\
\hline \multirow[t]{2}{*}{ Infant sex } & Male & $52 \cdot 0$ & 3411 & $36 \cdot 0$ & 1.00 & \\
\hline & Female & $48 \cdot 0$ & 3181 & $32 \cdot 7$ & 0.93 & $0.86,1.00$ \\
\hline \multirow[t]{2}{*}{ First child } & Not 1st & $58 \cdot 0$ & 4022 & 31.5 & 1.00 & \\
\hline & 1st & $42 \cdot 0$ & 2570 & 38.5 & 1.29 & $1.20,1.38$ \\
\hline
\end{tabular}

*Percentages are nationally representative with DHS weighted variable.

†This analysis included alive, singleton birth, infants under 24 months of age at time of survey.

¥Percentages are nationally representative with DHS weighted variable.

exempt from IRB review as no primary data were available from a deidentified data asset in the public domain.

Initially, descriptive statistics expressed as counts and frequencies were tabulated for all explanatory variables (Step 1). Next, unadjusted bivariate analyses examined the associations between the independent variable and each of the outcomes (Step 2). Variables associated with a $P<0.20$ were then entered into multivariable analyses examining each block of the hierarchical conceptual model (Step 3). Finally, variables associated with a $P<0.20$ in the within block analyses were entered as control variables in Step 4, i.e. multivariate hierarchical modelling. Blocks of variables were entered as following in the hierarchical modelling: (i) 'Household Variables'; (ii) 'Maternal Variables'; (iii) 'Health Systems Variables'; (iv) 'Child Variables'; (v) 'Early Feeding Environment Variables' (EBF model only) (Fig. 1). The associations between independent variables and outcomes were determined to be significant at the $5 \% P$-level. For both outcomes, within the healthcare system block we tested the interaction between C-section and number of prenatal visits (block 3) to find out if the simultaneous presence of both increased the risk of poor breastfeeding outcomes in a multiplicative ( $v$. additive) way.

\section{Results}

\section{Early breastfeeding initiation}

Table 1 shows the analytical sample characteristics and the prevalence of delayed breastfeeding initiation across 
Table 2 Final multiple hierarchical model to identify the factors associated with delayed breastfeeding initiation in children aged under 24 months ( $n$ 6592) (Colombia, DHS 2010)

\begin{tabular}{|c|c|c|c|}
\hline Variable & $\mathrm{PR}_{\text {adjusted }}$ & $95 \% \mathrm{Cl}$ & $P$ \\
\hline \multicolumn{4}{|l|}{ Model $1 \dagger$} \\
\hline \multicolumn{4}{|l|}{ Income quintile } \\
\hline Poorest & 1.00 & - & - \\
\hline Poorer & 1.15 & $1.04,1.27$ & $0.005^{\star}$ \\
\hline Middle & 1.20 & $1.08,1.34$ & $0.001^{* *}$ \\
\hline Richer & 1.22 & $1.08,1.38$ & $0.001^{* *}$ \\
\hline Richest & 1.28 & $1.11,1.48$ & $0.001^{\star *}$ \\
\hline \multicolumn{4}{|l|}{ Model $2 \ddagger$} \\
\hline \multicolumn{4}{|c|}{ Living with partner currently } \\
\hline Yes & 1.00 & - & - \\
\hline No & 0.91 & $0.83,1.00$ & $0.050^{*}$ \\
\hline \multicolumn{4}{|l|}{ Maternal education } \\
\hline Higher education & 1.00 & - & _- \\
\hline Lower education & 0.81 & $0.74,0.89$ & $<0.001^{* *}$ \\
\hline \multicolumn{4}{|c|}{ Maternal overweight/obese } \\
\hline No & 1.00 & - & - \\
\hline Yes & 1.09 & $1.01,1 \cdot 17$ & $0.031^{*}$ \\
\hline \multicolumn{4}{|l|}{ Pregnancy intention } \\
\hline Then & 1.00 & - & - \\
\hline Later & 1.03 & $0.94,1.12$ & 0.562 \\
\hline No More & 0.92 & $0.83,1.02$ & 0.115 \\
\hline \multicolumn{4}{|l|}{ Model $3 \S$} \\
\hline \multicolumn{4}{|l|}{$\begin{array}{l}\text { Skilled attendant at } \\
\text { birth }\end{array}$} \\
\hline Yes & 1.00 & - & - \\
\hline No & 1.09 & $1.01,1.18$ & $0.025^{\star}$ \\
\hline \multicolumn{4}{|l|}{ C-Section } \\
\hline No & 1.00 & - & - \\
\hline Yes & 2.08 & $1.92,2.25$ & $<0.001^{\star *}$ \\
\hline \multicolumn{4}{|l|}{$\begin{array}{l}\text { Number prenatal } \\
\text { visits }\end{array}$} \\
\hline $4-9$ & 1.00 & - & - \\
\hline $0-3$ & 0.98 & $0.88,1.11$ & 0.789 \\
\hline $10+$ & 1.15 & $1.00,1.32$ & 0.056 \\
\hline \multicolumn{4}{|l|}{ Model 4\| } \\
\hline \multicolumn{4}{|l|}{ Low birth weight } \\
\hline No & 1.00 & - & - \\
\hline Yes & 1.37 & $1.20,1.56$ & $<0.001^{\star *}$ \\
\hline \multicolumn{4}{|l|}{ Infant sex } \\
\hline Male & 1.00 & - & - \\
\hline Female & 0.97 & $0.89,1.06$ & 0.498 \\
\hline \multicolumn{4}{|l|}{ First child } \\
\hline Not 1st & 1.00 & - & - \\
\hline $1 \mathrm{st}$ & 1.23 & $1.12,1.35$ & $<0.001^{* *}$ \\
\hline
\end{tabular}

*Significant at $P<0.05{ }^{*}$ Significant at $P<0.001-$ reference category; no prevalence ratios or $\mathrm{Cl}$ calculated.

†Model 1: Income quintile and infant age.

¥Model 2: Model 1 + mother living with partner, maternal education, maternal overweight/obese and pregnancy intention.

§Model 3: Model 2 + skilled attendant at birth, C-section and number of prenatal visits ||Model 4: Model 3 + low infant birth weight, infant sex, and maternal primiparity.

outcome variables. The prevalence of EIBF within the first hour after birth was $66 \%$. The majority of respondents delivered in public health facilities $(95.2 \%)$ had at least a primary-level education (75.5\%), and $18.1 \%$ were adolescent mothers. The prevalence of C-section was $37.1 \%$ and $8.2 \%$ of infants had low birth weight. Proportionally more mothers who delivered via C-section ( $51.4 \%)$ did not practice EIBF relative to those who had a vaginal birth (24.6\%). There was a statistically significant interaction between $\mathrm{C}$-section and number of prenatal visits, the risk of delayed breastfeeding initiation was higher among those who had a C-section and had fewer than four visits $(O R=1.44 \mathrm{CI} 95 \%=1.20,1.72)$ or had ten visits or more $(\mathrm{OR}=1.50$, CI $95 \%=1.27,1.78)$ compared with those who had four to nine visits (data not shown).

Table 2 describes the results from the multivariate hierarchical modelling analysis by block. The modifiable risk factors associated with delayed breastfeeding initiation identified were: C-section delivery $(\mathrm{PR}=2 \cdot 08, \mathrm{CI}$ $95 \%=1.92, \quad 2.25)$, no skilled attendant at birth $(\mathrm{PR}=1.09, \quad \mathrm{CI} \quad 95 \%=1.01, \quad 1.18), \quad$ and maternal overweight/obesity $(\mathrm{PR}=1.09$, CI $95 \%=1.01,1 \cdot 17)$. Non-modifiable risk factors associated with delayed breastfeeding initiation identified were: maternal primiparity $(\mathrm{PR}=1 \cdot 23$, CI $95 \%=1 \cdot 12,1.35)$, infant low birth weight $(P R=1.37$, CI $95 \%=1.20,1.56)$, and higher household income quintile $(\mathrm{PR}=1 \cdot 20$, CI $95 \%=1.08$, 1.34). Mother not living with partner $(\mathrm{PR}=0.91$, CI $95 \%=0.83,1.00)$ and lower maternal education $(\mathrm{PR}=0.81$, CI $95 \%=0.74,0.89)$ were protective factors for EIBF. Supplementary material provides detailed information regarding the hierarchical EIBF models.

\section{Exclusive breastfeeding}

Table 3 shows the analytical sample characteristics ( $n$ 1512) and the prevalence of non-EBF across variables. A total of 688 infants (43\%) were exclusively breastfed through the first 6 months after birth. The majority of respondents were delivered in public health facilities (95.2\%), had at least a primary-level education (77.3\%) and were non-adolescent mothers between 20 to 49 years of age (76.6\%). The prevalence of C-section was $36.5 \%$ and $8.3 \%$ of infants had low birth weight. Around $60 \%$ had initiated breastfeeding within the first hour after birth and prelacteal feeds were used very frequent (62.4\%). There was a statistically significant interaction between C-section and number of prenatal visits, the risk of interrupting EBF was increased among those who had a $\mathrm{C}$-section and had ten visits or more $(\mathrm{OR}=1.24$, CI $95 \%=1.03,1.51)$ compared with those who had four to nine visits (data not shown).

Table 4 describes the results from the multivariate hierarchical modelling analysis by block. The independent modifiable risk factors identified for EBF interruption were: C-section delivery $(\mathrm{PR}=1 \cdot 12$, CI $95 \%=1.02$, 1.23) and prelacteal feeding $(P R=1 \cdot 51$, CI $95 \%=1.37$, 1.68). Non-pregnancy intention was a protective factor against $\mathrm{EBF}$ interruption $(\mathrm{PR}=0.82$, CI $95 \%=0.72$, 0.93). The non-modifiable risk factor associated with $\mathrm{EBF}$ interruption was higher household income quintile $(\mathrm{PR}=1 \cdot 23$, CI $95 \%=1 \cdot 08,1 \cdot 40)$. Supplementary material provides detailed information regarding the EBF hierarchical models. 
Table 3 Descriptive characteristics of analytical sample $(n$ 1512) and bivariate analyses of EBF interruption among children aged under 6 months and unadjusted prevalence ratios (Demographic Health Surveys, Colombia 2010)

\begin{tabular}{|c|c|c|c|c|c|c|}
\hline & & \multicolumn{2}{|c|}{ Analytical sample } & \multicolumn{3}{|c|}{ Exclusive breastfeeding interruption } \\
\hline & & $\%^{*}$ & $n \dagger$ & $\% \ddagger$ & Non-adjusted PR & $95 \% \mathrm{Cl}$ \\
\hline \multirow{2}{*}{ Exclusive breastfeeding $<6$ months } & Yes & $43 \cdot 0$ & 688 & - & - & - \\
\hline & No & $57 \cdot 0$ & 824 & - & - & - \\
\hline \multicolumn{7}{|l|}{ Distal block (1): household variables } \\
\hline \multirow[t]{2}{*}{ Place residence } & Urban & $72 \cdot 8$ & 951 & 56 & 1.00 & \\
\hline & Rural & $27 \cdot 2$ & 561 & 59.5 & 0.90 & $0.81,1.00$ \\
\hline \multirow[t]{5}{*}{ Income quintile } & Poorest & $25 \cdot 3$ & 563 & 59 & 1.00 & \\
\hline & Poorer & $23 \cdot 0$ & 416 & $57 \cdot 1$ & $1 \cdot 21$ & $1.07,1.37$ \\
\hline & Middle & 23.5 & 281 & $59 \cdot 3$ & $1 \cdot 20$ & $1.04,1.37$ \\
\hline & Richer & 18.4 & 170 & $51 \cdot 8$ & 1.14 & $0.97,1.35$ \\
\hline & Richest & 9.9 & 82 & $55 \cdot 3$ & 1.26 & $1.03,1.53$ \\
\hline \multirow[t]{2}{*}{ Number adults in household } & $1-2$ & $27 \cdot 7$ & 407 & $49 \cdot 6$ & 1.00 & \\
\hline & \multicolumn{5}{|c|}{ Intermediate block (2): maternal variables } & $0.98,1.22$ \\
\hline \multirow[t]{2}{*}{ Living with partner currently } & Yes & 73.9 & 1161 & $55 \cdot 4$ & $1 \cdot 00$ & \\
\hline & No & $26 \cdot 1$ & 351 & 61.4 & 1.12 & $1.01,1.25$ \\
\hline \multirow[t]{2}{*}{ Education level } & Higher education & $77 \cdot 3$ & 1071 & $56 \cdot 2$ & 1.00 & \\
\hline & Lower education & $22 \cdot 7$ & 441 & 59.5 & 0.91 & $0.82,1.02$ \\
\hline \multirow[t]{2}{*}{ Maternal overweight/obese } & No & 54.9 & 664 & $60 \cdot 3$ & 1.00 & \\
\hline & Yes & $45 \cdot 2$ & 560 & $63 \cdot 1$ & 1.06 & $0.96,1.17$ \\
\hline \multirow{2}{*}{ Adolescent mother $(\leq 19)$} & No & $76 \cdot 6$ & 1158 & 55.5 & 1.00 & \\
\hline & Yes & 23.5 & 354 & $61 \cdot 6$ & 1.05 & $0.94,1.17$ \\
\hline \multirow[t]{3}{*}{ Pregnancy intention } & Then & $48 \cdot 1$ & 704 & $56 \cdot 3$ & 1.00 & \\
\hline & Later & $31 \cdot 1$ & 451 & $59 \cdot 8$ & 1.01 & $0.91,1.12$ \\
\hline & No more & $20 \cdot 8$ & 357 & 54.2 & 0.85 & $0.75,0.97$ \\
\hline \multirow[t]{2}{*}{ Working mother } & No & 71.6 & 1058 & 55.2 & 1.00 & \\
\hline & Yes & $28 \cdot 4$ & 454 & 61.4 & 1.01 & $0.92,1.12$ \\
\hline \multicolumn{7}{|l|}{$\begin{array}{l}\text { Intermediate block (3): health systems } \\
\text { variables }\end{array}$} \\
\hline \multirow[t]{2}{*}{ Skilled attendant at birth } & Yes & 39.9 & 583 & $60 \cdot 7$ & 1.00 & \\
\hline & No & $60 \cdot 1$ & 929 & 54.5 & 0.91 & $0.83,1.00$ \\
\hline \multirow{2}{*}{ Delivery in public health facility } & Yes & $95 \cdot 2$ & 1353 & $57 \cdot 2$ & 1.00 & \\
\hline & No & 4.8 & 159 & 53.0 & 0.69 & $0.56,0.86$ \\
\hline \multirow{2}{*}{ C-Section } & No & 63.5 & 1029 & $55 \cdot 8$ & 1.00 & \\
\hline & Yes & 36.5 & 483 & 58.9 & 1.16 & $1.05,1.27$ \\
\hline \multirow[t]{3}{*}{ Number prenatal visits } & $4-9$ & $78 \cdot 4$ & 1106 & $58 \cdot 1$ & 1.00 & \\
\hline & $0-3$ & $12 \cdot 9$ & 295 & 51.9 & 0.77 & $0.66,0.88$ \\
\hline & $10+$ & $8 \cdot 7$ & 100 & 54.7 & 1.03 & $0.87,1.23$ \\
\hline \multicolumn{7}{|l|}{ Proximal block (4): child variables } \\
\hline \multirow[t]{2}{*}{ Low birth weight } & No & $91 \cdot 7$ & 1147 & $56 \cdot 8$ & 1.00 & \\
\hline & Yes & $8 \cdot 3$ & 81 & $40 \cdot 6$ & 0.77 & $0.60,1.01$ \\
\hline Infant sex & Male & $53 \cdot 1$ & 799 & 59.5 & 1.00 & \\
\hline & Female & $47 \cdot 0$ & 713 & 54 & 0.92 & $0.84,1.01$ \\
\hline First child & Not 1st & $57 \cdot 6$ & 923 & $55 \cdot 3$ & 1.00 & \\
\hline & $1 \mathrm{st}$ & $42 \cdot 4$ & 589 & 59.2 & $1 \cdot 10$ & $1.00,1.21$ \\
\hline $\begin{array}{l}\text { Proximal block (5): early feeding environm } \\
\text { variables (only for EBF) }\end{array}$ & & & & & & \\
\hline Prelacteal feeds & No & $62 \cdot 4$ & 968 & $49 \cdot 1$ & 1.00 & \\
\hline & Yes & $37 \cdot 6$ & 528 & $69 \cdot 1$ & 1.57 & $1.44,1.72$ \\
\hline Early breastfeeding initiation (within $1 \mathrm{~h}$ ) & Yes & 60.5 & 972 & $56 \cdot 2$ & 1.00 & \\
\hline & No & 39.5 & 524 & $57 \cdot 3$ & 1.08 & $0.98,1.19$ \\
\hline
\end{tabular}

*Percentages are nationally representative with DHS weighted variable.

†This analysis included alive, singleton birth, infants under 6 months of age at time of survey.

$\ddagger$ Percentages are nationally representative with $\mathrm{DHS}$ weighted variable.

\section{Discussion}

To our knowledge, this is the first study to investigate determinants of breastfeeding practices in Colombia looking at modifiable risk factors from a healthcare system perspective. C-section, lack of skilled attendant at birth, prelacteal feeding, and maternal overweight/obesity were the identified modifiable risk factors that can be integrated and addressed through a package of health system interventions to improve breastfeeding outcomes in Colombia. Evidence has shown that designing tailored interventions focusing on multiple relevant risk factors increases breastfeeding rates, particularly in non-high income countries such as Colombia $^{(1,28)}$. Strategies that promote breastfeeding and engage individuals, facilities, and communities in interventions that integrate education and support for optimal 
Table 4 Final multiple hierarchical model to identify the factors associated with interrupting EBF in children aged under 6 months (n 1512) (Colombia, DHS 2010)

\begin{tabular}{|c|c|c|c|}
\hline Variable & PR adjusted & $95 \% \mathrm{Cl}$ & $P$ \\
\hline \multicolumn{4}{|l|}{ Model 1† } \\
\hline \multicolumn{4}{|c|}{ Income quintile } \\
\hline Poorest & 1.00 & - & - \\
\hline Poorer & $1 \cdot 21$ & $1.07,1.35$ & $0.002^{*}$ \\
\hline Middle & 1.23 & $1.08,1.40$ & $0.002^{*}$ \\
\hline Richer & 1.17 & $1.00,1.36$ & $0.049^{*}$ \\
\hline Richest & 1.26 & $1.04,1.52$ & $0.019^{*}$ \\
\hline \multicolumn{4}{|c|}{ Number adults in } \\
\hline $1-2$ & 1.00 & - & - \\
\hline $3+$ & 1.11 & $1.00,1.23$ & 0.054 \\
\hline \multicolumn{4}{|l|}{ Model $2 \ddagger$} \\
\hline \multicolumn{4}{|c|}{$\begin{array}{l}\text { Living with partner } \\
\text { currently }\end{array}$} \\
\hline Yes & 1.00 & _- & _ \\
\hline No & $1 \cdot 10$ & $0.99,1.23$ & 0.079 \\
\hline \multicolumn{4}{|c|}{ Maternal overweight/obese } \\
\hline No & 1.00 & - & - \\
\hline Yes & 1.05 & $0.96,1.15$ & 0.294 \\
\hline \multicolumn{4}{|c|}{ Pregnancy intention } \\
\hline Then & 1.00 & - & - \\
\hline Later & 0.99 & $0.89,1 \cdot 10$ & 0.812 \\
\hline No More & 0.82 & $0.72,0.93$ & $0.003^{*}$ \\
\hline \multicolumn{4}{|l|}{ Model $3 \S$} \\
\hline \multicolumn{4}{|c|}{$\begin{array}{l}\text { Delivery in public health } \\
\text { facility }\end{array}$} \\
\hline Yes & 1.00 & - & - \\
\hline No & 0.87 & $0.70,1.08$ & 0.213 \\
\hline \multicolumn{4}{|l|}{ C-Section } \\
\hline No & 1.00 & - & - \\
\hline Yes & $1 \cdot 12$ & $1.02,1.23$ & $0.019^{\star}$ \\
\hline \multicolumn{4}{|c|}{ Number prenatal visits } \\
\hline $4-9$ & 1.00 & - & - \\
\hline $0-3$ & 0.87 & $0.75,1.02$ & 0.086 \\
\hline $10+$ & 1.04 & $0.88,1.25$ & 0.625 \\
\hline \multicolumn{4}{|l|}{ Model 4\| } \\
\hline \multicolumn{4}{|c|}{ Low birth weight } \\
\hline No & 1.00 & - & - \\
\hline Yes & 0.79 & $0.60,1.04$ & 0.089 \\
\hline \multicolumn{4}{|l|}{ Infant sex } \\
\hline Male & 1.00 & _- & - \\
\hline Female & 0.93 & $0.83,1.04$ & 0.192 \\
\hline \multicolumn{4}{|l|}{ First child } \\
\hline Not 1st & 1.00 & _- & - \\
\hline 1 st & 1.01 & $0.90,1.14$ & 0.876 \\
\hline \multicolumn{4}{|l|}{ Model 59} \\
\hline \multicolumn{4}{|c|}{ Prelacteal feeds } \\
\hline No & 1.00 & _ & - \\
\hline Yes & 1.51 & $1.37,1.68$ & $<0.001^{* *}$ \\
\hline
\end{tabular}

*Significant at $P<0.05{ }^{* *}$ Significant at $P<0.001-$ reference category; no prevalence ratios or $\mathrm{Cl}$ calculated.

tModel 1: income quintile, number of adults in household, and infant age.

¥Model 2: Model 1 + mother living with partner, maternal overweight/obese, and pregnancy intention.

§Model 3: Model $2+$ delivery in public health facility, C-section, and number of prenatal visits.

||Model 4: Model $3+$ low infant birth weight, infant sex, and maternal primiparity. qModel 5: Model $3+$ prelacteal feeds.

breastfeeding practices at multiple levels have been successful in improving breastfeeding indicators and achieving country-level goals ${ }^{(1,28,29)}$.

In our study, C-section was a risk factor for both delayed breastfeeding initiation and interruption of EBF. C-section is often associated with obstetric complications that may lead to mother-newborn separation, which in turn can reduce the likelihood of EIBF and the duration of any breastfeeding or $\mathrm{EBF}^{(30-32)}$. The prevalence of C-section is higher in Latin America relative to other low-and-middle income (LMIC) regions, accounting for $24-45 \%$ of births in public and private sector facilities ${ }^{(33,34)}$. This high prevalence combined with the dearth of skilled birth attendants found in our study points to a key barrier for EIBF. Skilled attendants at birth, particularly those who are properly trained in supporting optimal breastfeeding practices, are shown to improve maternal EIBF as well as longer-term feeding practices ${ }^{(35)}$. Therefore, it is important to acknowledge that addressing barriers for timely breastfeeding initiation is a crucial first step for achieving longer term EBF and any breastfeeding success $^{(32,36,37)}$. Additionally, consistent evidence from countries across world regions has identified C-section as a risk factor for influencing pre-lacteal feeding ${ }^{(38)}$. Indeed, Boccolini et al. analysed data from Latin America and the Caribbean, including Colombia and found important inequities between C-section delivery and risk of prelacteal feeds. Specifically, women of lower socio-economic status experienced a heightened risk of milk-based prelacteal feeding associated with C-section delivery ${ }^{(38)}$. Pre-lacteal feeding is of serious concern as it has consistently been identified as a risk factor for EBF in cohort and cross-sectional studies globally ${ }^{(39)}$. These findings combined with the high rates of hospital or clinic births (95.6\%) in Colombia indicate an opportunity for improving healthcare system support and protection of breastfeeding mothers in the perinatal and postnatal period in Colombia.

Maternal pregnancy intention is generally described in the literature as associated with positive breastfeeding behaviour as compared with lack of intention at the time of conception ${ }^{(40)}$. However, in our study, nonpregnancy intention was associated with longer EBF perhaps because, in the Colombian context, women with unplanned pregnancies may have received more attention and support with regards to their breastfeeding plans. More than $50 \%$ of pregnancies are not planned ${ }^{(41)}$, thus, providing breastfeeding education during the antenatal care is key to encouraging non-intentional pregnant women to breastfeed. A patient-centred approach to such promotion activities, such as incorporating pregnancy intention into counselling, is key to achieving optimally targeted and effective interventions ${ }^{(42,43)}$. Maternal overweight/obesity is a recognized risk factor for delayed breastfeeding initiation and is also implicated in shorter overall breastfeeding duration ${ }^{(44,45)}$ owing to biological, mechanical, behavioural and/or psychological factors. This characteristic is of particular relevance in the global context as prevalence of obesity is increasing worldwide and in LMIC such as Colombia ${ }^{(46)}$. Providing timely support and counselling for obese and overweight women is crucial to improving both $\mathrm{EIBF}$ and $\mathrm{EBF}^{(47)}$. 
Several non-modifiable factors associated with suboptimal breastfeeding were found in our study. The finding of increased EBF interruption with higher income quintiles is consistent with findings from other LMIC $^{(1)}$. Indeed, in these countries poorer women are more likely to initiate and continue breastfeeding for longer periods of time as compared with their richer counterparts ${ }^{(1)}$. Consistent with our findings, infant low birth weight and primiparity have been previously found to be associated with suboptimal breastfeeding practices ${ }^{(31,48)}$. Lower maternal education and mother not living with partner were protective factors to delayed breastfeeding initiation in Colombia, which is somewhat consistent with some but not all studies previously conducted ${ }^{(49,50)}$.

It is important to acknowledge that Colombia has been working for several years towards building an enabling breastfeeding environment. In 2010, a 'Ten Year Plan' (2010-2020) was formulated to strengthen breastfeeding programmes ${ }^{(7,51)}$. Universal maternity leave up to 18 -weeks and maternal workplace protection strategies were recently legislated ${ }^{(52,53)}$. However, our findings clearly indicate that there are key modifiable risk factors that can be addressed through healthcare systems interventions. Indeed, the modifiable factors identified in this study can inform the development of a package of interventions to strengthen the coordination of efforts on breastfeeding counselling and support, including enhancing BFHI implementation $^{(5,6)}$ and breastfeeding counselling within the Colombian health systems ${ }^{(1,22)}$. Evidence has shown that these identified factors are amenable to change and improvement through clear and specific recommendations and interventions to better support breastfeeding practices, underscoring the strong focus needed on the healthcare systems $^{(54-56)}$.

Our findings must be carefully interpreted due to the cross-sectional survey design, precluding the establishment of the temporality of associations. The retrospective nature of the surveying method also introduces potential recall bias. The external validity of the study was tested and both analytical samples were similar for most household and maternal variables analysed, differing only slightly in place of residence, marital status, and maternal age. However, these characteristics were not associated with both outcomes; therefore, we believe that this limitation did not significantly affect the external validity of the study. It is also important to note that the study data were from 2010; nevertheless, our literature review indicates that the findings are still very applicable to the current breastfeeding context in Colombia and can be used to generate hypotheses to be tested through future quasi-experimental and experimental studies ${ }^{(19)}$.

In conclusion, women in Colombia need additional breastfeeding support at multiple levels to engage in optimal infant feeding practices. The identification of modifiable risk factors for EIBF and EBF can help inform the development of a tailored package of interventions to improve the coverage and quality of breastfeeding counselling through the healthcare system in Colombia.

\section{Acknowledgements}

Acknowledgements: Not applicable. Financial support: This research received no specific grant from any funding agency, commercial or not-for-profit sectors. No other entity besides the authors had a role in the design, analysis, or writing of this article. Conflicts of interest: None. Authorship: S.F. was responsible for formulating the research question, analysing the data, and writing the article with advice, reviewing, and editing from R.P.-E. and G.B. Ethics of buman subject participation: This research did not involve human subjects but relied on secondary data analysis of a deidentified dataset. Ethical standards were upheld in the research process.

\section{Supplementary material}

To view supplementary material for this article, please visit https://doi.org/10.1017/S1368980019002180

\section{References}

1. Victora CG, Bahl R, Barros AJ, et al. (2016) 1 Breastfeeding in the 21st century: epidemiology, mechanisms, and lifelong effect. Lancet 387, 475-490.

2. Hansen K (2016) Breastfeeding: a smart investment in people and in economies. Lancet 387, 416.

3. Ochoa Parra G \& Casallas Murillo AL (2014) Report on the Situation of Infant and Young Child Feeding in Colombia. The Committee on the Rights of the Child. International Baby Food Action Network (IBFAN).

4. Organización Panamericana de la Salud (2011) 30 años del Código en América Latina: Un recorrido sobre diversas experiencias de aplicación del Código Internacional de Comercialización de Sucedáneos de la Leche Materna en la Región entre 1981 y 2011.

5. Perez-Escamilla R (2007) Evidence based breast-feeding promotion: the Baby-Friendly Hospital Initiative. J Nutr 137, 484-487.

6. UNICEF (2005) Instituciones Amigas de la Mujer y la Infancia en el Marco de los Derechos: Manual para su aplicación por el interés superior del Niño, pp. 59-60.

7. Organizacion Panamericana de la Salud (2013) Avances y retos de la lactancia materna en Colombia 1970 a 2013: un recorrido sobre 40 años de experiencias en la aplicación de politicas públicas. Washington, DC: Organización Panamericana de la Salud.

8. Escobar M-L, Giedion U, Giuffrida A, et al. (2009) Colombia: after a decade of health system reform. From Few to Many 1.

9. World Breastfeeding Trends Initiative (2016) Colombia 2016 Estado de la "estrategia mundial para la alimentación del lactante niño pequeño" [Iniciativa Mundial sobre Tendencias de la Lactancia Materna (WBTi), editor]. San José, Costa Rica: World Breastfeeding Trends Initiative.

10. World Breastfeeding Trends Initiative (2016) Seguimiento WBTi 2016 a 2017: Colombia 2017. Iniciativa munidal sobre tendencias de la lactancia materna. Bogotá, Colombia: 
International Baby Food Action Network (IBFAN): Coordinacion Nacional Grupo IBFAN Colombia.

11. Departamento Administrativo Nacional de Estadística (2015) Encuesta Nacional de Calidad de Vida - ECV 2015. Available from: https://www.dane.gov.co/files/ investigaciones/condiciones_vida/calidad_vida/Boletin_ Tecnico_ECV_2015.pdf

12. Profamilia/Colombia (2011) Colombia Encuesta Nacional de Demografía y Salud 2010. Bogotá: Profamilia, Ministerio de la Protección Social Republica de Colombia, and USAID.

13. UNICEF (2017) Statistics: Colombia. Available from: http:// www.who.int/topics/breastfeeding/en/

14. Ogbo FA, Page A, Idoko J, et al. (2018) Population attributable risk of key modifiable risk factors associated with non-exclusive breastfeeding in Nigeria. BMC Public Health 18, 247.

15. Lutter CK \& Morrow AL (2013) Protection, promotion, and support and global trends in breastfeeding. Adv Nutr $\mathbf{4}$, 213-219.

16. Pan American Health Organization (2012) Colombia. Available from: http://www.paho.org/salud-en-las-americas2012/index.php?option $=$ com_docman $\& v i e w=$ download $\&$ category_slug=hia-2012-country-chapters-22\&alias $=123$ colombia-123\&Itemid $=231 \&$ lang $=$ en

17. World Health Organization (2010) Indicators for assessing infant and young child feeding practices: part 1 \& 2 definitions \& measurement. Geneva: WHO.

18. World Health Organization (2018) Nutrition: Breastfeeding. Geneva: WHO.

19. Victora CG, Huttly SR, Fuchs SC, et al. (1997) The role of conceptual frameworks in epidemiological analysis: a hierarchical approach. Int J Epidemiol 26, 224-227.

20. Boccolini CS, Carvalho ML \& Oliveira MI (2015) Factors associated with exclusive breastfeeding in the first six months of life in Brazil: a systematic review. Rev Saude Publica 49, 91

21. Venancio SI \& Monteiro CA (2006) Individual and contextual determinants of exclusive breast-feeding in São Paulo, Brazil: a multilevel analysis. J Public Health Nutr 9, $40-46$.

22. Rollins NC, Bhandari N, Hajeebhoy N, et al. (2016) Why invest, and what it will take to improve breastfeeding practices? Lancet 387, 491-504.

23. Garret DA, Sanga JK, Kothari MT, et al. (2011) Field-friendly techniques for assessment of biomarkers of nutrition for development. Am J Clin Nutr 94, 685S-690S.

24. Osorio AM, Tovar LM \& Rathmann K (2014) Individual and local level factors and antenatal care use in Colombia: a multilevel analysis. J Cad Saude Publica 30, 1079-1092.

25. Carter EB, Tuuli MG \& Caughey AB, et al. (2016) Number of prenatal visits and pregnancy outcomes in low-risk women. $J$ Perinatol 36, 178.

26. StataCorp LLC. (2019) Mutlilevel generalized linear models. Available from https://www.stata.com/features/overview/ multilevel-generalized-linear-models/

27. Zou G (2004) A modified Poisson regression approach to prospective studies with binary data. Am J Epidemiol 159, 702-706.

28. Haroon S, Das JK, Salam RA, et al. (2013) Breastfeeding promotion interventions and breastfeeding practices: a systematic review. BMC Public Health 13, S20.

29. Sinha B, Chowdhury R, Sankar MJ, et al. (2015) Interventions to improve breastfeeding outcomes: a systematic review and meta-analysis. J Acta Paediatrica 104, 114-134.

30. Rowe-Murray HJ \& Fisher JR (2002) Baby friendly hospital practices: cesarean section is a persistent barrier to early initiation of breastfeeding. Birth 29, 124-131.

31. Chapman DJ \& Perez-Escamilla R (1999) Identification of risk factors for delayed onset of lactation. J Am Diet Assoc 99, 450-454.
32. Cato K, Sylvén SM, Lindbäck J, et al. (2017) Risk factors for exclusive breastfeeding lasting less than two monthsIdentifying women in need of targeted breastfeeding support. PLoS One 12, e0179402.

33. Benova L, Macleod D, Footman K, et al. (2015) Role of the private sector in childbirth care: cross-sectional survey evidence from 57 low-and middle-income countries using demographic and health surveys. Trop Med Int Health 20, 1657-1673.

34. Oakley L, Benova L, Macleod D, et al. (2018) Early breastfeeding practices: descriptive analysis of recent demographic and health surveys. Matern Child Nutr 14, e12535.

35. Selim L (2018) Breastfeeding from the first hour of birth: What works and what hurts. New York: UNICEF.

36. Esteves TMB, Daumas RP, Oliveira MI, et al. (2014) Factors associated to breastfeeding in the first hour of life: systematic review. Rev Saude Publica 48, 697-708.

37. DiGirolamo AM, Grummer-Strawn LM \& Fein S (2001) Maternity care practices: implications for breastfeeding. Birth 28, 94-100.

38. Boccolini CS, Pérez-Escamilla R, Giugliani ERJ, et al. (2015) Inequities in milk-based prelacteal feedings in Latin America and the Caribbean: the role of cesarean section delivery. J Hum Lact 31, 89-98.

39. Pérez-Escamilla $\mathrm{R}$, Segura-Millán $\mathrm{S}$, Canahuati $\mathrm{J}$, et al. (1996) Prelacteal feeds are negatively associated with breast-feeding outcomes in Honduras. J Nutr 126, $2765-2773$.

40. Keddem S, Frasso R, Dichter M, et al. (2018) The association between pregnancy intention and breastfeeding. J Hum Lact 34, 97-105.

41. Sedgh G, Singh S \& Hussain R (2014) Intended and unintended pregnancies worldwide in 2012 and recent trends. Stud Fam Plann 45, 301-314.

42. Taylor JS \& Cabral H (2002) Are women with an unintended pregnancy less likely to breastfeed? J Fam Pract 51, 431-437.

43. Murray EK, Ricketts S \& Dellaport J (2007) Hospital practices that increase breastfeeding duration: results from a population-based study. Birth 34, 202-211.

44. Amir LH \& Donath S (2007) A systematic review of maternal obesity and breastfeeding intention, initiation and duration. BMC Pregnancy Childbirth 7, 9.

45. Wojcicki JM (2011) Maternal prepregnancy body mass index and initiation and duration of breastfeeding: a review of the literature. J Women Health 20, 341-347.

46. Hossain P, Kawar B \& El Nahas M (2009) Obesity and diabetes in the developing world-a growing challenge. $N$ Engl J Med 356, 213-215.

47. Claesson M, Larsson L, Steen L, et al. (2018) "You just need to leave the room when you breastfeed" breastfeeding experiences among obese women in Sweden-a qualitative study. BMC Pregnancy Childb 18, 39.

48. Patil CL, Turab A, Ambikapathi R, et al. (2015) Early interruption of exclusive breastfeeding: results from the eight-country MAL-ED study. J Health Popul Nutr 34, 10.

49. Patel A, Bucher S, Pusdekar Y, et al. (2015) Rates and determinants of early initiation of breastfeeding and exclusive breast feeding at 42 days postnatal in six low and middleincome countries: a prospective cohort study. Reprod Health 12, S10

50. Dewey KG, Nommsen-Rivers LA, Heinig MJ, et al. (2003) Risk factors for suboptimal infant breastfeeding behavior, delayed onset of lactation, and excess neonatal weight loss. Pediatrics 112, 607-619.

51. Betancourt DP (2010) Plan Decenal Lactancia Materna 20102020. Bogota, Colombia: Ministerio de la Protección Social.

52. Congreso de Colombia (2017) Ley No 1823. Available from: http://es.presidencia.gov.co/normativa/normativa/LEY\% 201823\%20DEL\%204\%20DE\%20ENERO\%20DE\%202017.pdf 
53. Secretaría Jurídica Distrital (2017) Proyecto de Acuerdo 422 de 2017: Por el cual se estableen estrategias para la protección, promoción, fomento y apoyo a la cultura de la lactancia materna en el Distrito Capital. Concejo de Bogotá D.C.

54. World Health Organization (1989) Protecting, promoting and supporting breast-feeding: the special role of maternity services. Geneva: WHO.
55. Lamprea E \& García J (2016) Closing the gap between formal and material health care coverage in Colombia. Health Human Rights 2, 49-65.

56. Perez-Escamilla R \& Engmann C. (2019) Integrating nutrition services into health care systems platforms. Where are we and where do we go from here. Matern Child Nutr 15, 1-5. 